\title{
Spontaneous and deliberate creative cognition during and after psilocybin exposure
}

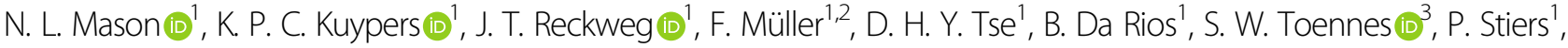 \\ A. Feilding ${ }^{4}$ and J. G. Ramaekers (1) ${ }^{1}$
}

\begin{abstract}
Creativity is an essential cognitive ability linked to all areas of our everyday functioning. Thus, finding a way to enhance it is of broad interest. A large number of anecdotal reports suggest that the consumption of psychedelic drugs can enhance creative thinking; however, scientific evidence is lacking. Following a double-blind, placebo-controlled, parallel-group design, we demonstrated that psilocybin $(0.17 \mathrm{mg} / \mathrm{kg})$ induced a time- and construct-related differentiation of effects on creative thinking. Acutely, psilocybin increased ratings of (spontaneous) creative insights, while decreasing (deliberate) task-based creativity. Seven days after psilocybin, number of novel ideas increased. Furthermore, we utilized an ultrahigh field multimodal brain imaging approach, and found that acute and persisting effects were predicted by within- and between-network connectivity of the default mode network. Findings add some support to historical claims that psychedelics can influence aspects of the creative process, potentially indicating them as a tool to investigate creativity and subsequent underlying neural mechanisms. Trial NL6007; psilocybin as a tool for enhanced cognitive flexibility; https://www.trialregister.nl/trial/6007.
\end{abstract}

\section{Introduction}

Creativity is an essential cognitive ability linked to all areas of our everyday life, allowing us to adapt to an everchanging environment and come up with ways to solve problems. Although arguably difficult to define, the creative process has been viewed as a dynamic process ${ }^{1,2}$, requiring shifting between different modes of thought in order to reach an end result ${ }^{3}$. These modes include divergent thinking (DT), which consists of generating novel and original ideas, and convergent thinking (CT), the subsequent evaluation of generated ideas in regards to their usefulness and effectiveness ${ }^{4,5}$. Importantly, as well as being an essential process for everyday functioning, the (in)ability to think "outside of the box" has also been associated with psychological disorders, such as

\footnotetext{
Correspondence: N L. Mason (natasha.mason@maastrichtuniversity.nl) or J G. Ramaekers (j.ramaekers@maastrichtuniversity.nl)

'Department of Neuropsychology and Psychopharmacology, Faculty of Psychology and Neuroscience, Maastricht University, P.O. Box 616, 6200 MD Maastricht, the Netherlands

${ }^{2}$ Department of Psychiatry (UPK), University of Basel, Basel, Switzerland

Full list of author information is available at the end of the article
}

depression and anxiety ${ }^{6,7}$. Here, it has been regarded both as a transdiagnostic process, i.e., a risk or maintaining factor shared across disorders, and as a potential therapeutic intervention, promoting adaptive interpretations and coping strategies ${ }^{7,8}$. Thus taken together, finding a way to enhance creative thinking is of broad interest among multiple disciplines, ranging from industry to psychopathology.

Over the years, a number of anecdotal reports have accumulated suggesting that the consumption of serotonin (5-HT) 2A agonist psychedelic drugs ${ }^{9}$, like lysergic acid diethylamide (LSD), psilocybin, and mescaline, can enhance creativity ${ }^{10,11}$. Famous examples of psychedelicaffiliated creative breakthroughs span the fields of science, technology, and art; including Kary Mullis' discovery of the polymerase chain reaction, the 1960's California-based computer industry, and the literary works of authors, such as Aldous Huxley and Ken Kesey ${ }^{10,11}$. That said, although there has been much historical interest in the ability of psychedelics to enhance creative capacity, the scientific literature is largely lacking. Initial scientific studies into

\section{(c) The Author(s) 2021}

(c) (i) Open Access This article is licensed under a Creative Commons Attribution 4.0 International License, which permits use, sharing, adaptation, distribution and reproduction cc) in any medium or format, as long as you give appropriate credit to the original author(s) and the source, provide a link to the Creative Commons license, and indicate if changes were made. The images or other third party material in this article are included in the article's Creative Commons license, unless indicated otherwise in a credit line to the material. If material is not included in the article's Creative Commons license and your intended use is not permitted by statutory regulation or exceeds the permitted use, you will need to obtain permission directly from the copyright holder. To view a copy of this license, visit http://creativecommons.org/licenses/by/4.0/. 
the effects of psychedelics on creativity date as far back as the 1950's, and ran until the drugs were banned in the United States in the late 1960s (refs. ${ }^{10,11}$ ). However, findings of these studies were largely inconclusive, and limited in regards to present-day methodology ${ }^{12-14}$. Instead, preliminary contemporary work provides stronger indications that psychedelics can affect creativityrelated constructs ${ }^{1,15-19}$, with phenomenological reports suggesting psychedelics induce a hyper-associative state of cognition $^{1}$, and pseudo-experimental studies finding acute $^{20}$ and subacute ${ }^{21-23}$ increases in DT or CT after consumption of a psychedelic in a naturalistic setting. Overall, evidence suggests that psychedelics can mediate changes in particular constructs of creativity, however direct, experimental evidence is lacking.

Importantly, if psychedelics can mediate changes in particular dimensions of the creative process, they could be used as a potentially novel tool to investigate these processes ${ }^{1}$, as well as the underlying neural mechanisms $^{24}$. In regards to creative cognition, previous research has consistently implicated the coordination of three resting-state networks (RSNs); the default mode network (DMN), suggested to support idea generation, the frontoparietal control network (FPN), supporting idea evaluation, and areas of the salience network ( $\mathrm{SN}$ ), suggested to facilitate the shift between the internally (DMN) and externally (FPN) oriented cognitive networks ${ }^{25-27}$. Although usually opposing neural processes, the DMN and FPN have been found to interact during creative cognition, suggesting a dynamic shift between these networks facilitates the shift between idea generation and evaluation across time ${ }^{25}$. Recently, this shift between neurocognitive dissociable states has been further conceptualized into a new theory (the dynamic framework of thought ${ }^{1,28}$ ), suggesting that creative thought is an alternation between states with low (DMN driven) to high (FPN driven) cognitive constraints, operating on mnemonic information from the medial temporal region. Interestingly, the authors incorporated the psychedelic state into this model $^{1}$, suggesting that psychedelics represent a state of unconstrained cognition, with neural evidence stemming from previous imaging studies which suggest they acutely induce a flexible brain state ${ }^{29-31}$, characterized by an acute disintegration of normally highly organized activity within $\mathrm{RSNs}^{32-36}$, a simultaneous widening of dynamic repertoires of connectivity states $^{37}$, and increased coupling of RSNs that are usually anticorrelated $^{34,35,38-40}$. Changes in connectivity have also been found to outlast the acute stage, with reports of increased within-network DMN connectivity 1-day postdrug administration ${ }^{41-43}$, and increased between-network connectivity up to 1 -month post-drug administration ${ }^{44,45}$.

Overall, previous research suggests an acute and even persisting effect of psychedelics on creativity-related cognition and neural mechanisms. However, no study has yet directly assessed the (sub)acute effect of a psychedelic on the two constructs of creativity (DT and CT) in a controlled trial, or its relationship to drug-induced brain changes. Therefore, the aim of this study was twofold: to assess the acute and persisting (7-day post-drug administration) effect of the classic psychedelic, psilocybin, on divergent and CT; and to assess (acute) biological predictors of changes in these constructs. To do this, divergent and CT were quantified via two widely used cognitive tasks, the alternate uses test $(\mathrm{AUT})^{46}$ and the picture concept test (PCT) ${ }^{20}$. As evidence suggests a neurocognitive distinction between deliberate (task-based) creative cognition and spontaneous (insight) creative $\operatorname{cognition}^{47,48}$, subjective feelings of spontaneous creative thinking were also assessed, via a well-established altered states of consciousness questionnaire ${ }^{49}$. Then, the relationship between cognitive and subjective ratings of creativity, within- and between-network connectivity of the $\mathrm{DMN}, \mathrm{FPN}$, and $\mathrm{SN}$, and glutamate concentrations in the medial prefrontal cortex (mPFC) and hippocampus, were evaluated. The latter was of interest as evidence suggests that network connectivity changes during the psychedelic state may result from changes in local network integrity due to downstream stimulation of glutamatergic signaling ${ }^{43,50,51}$, this is of particular interest in the mPFC and hippocampus due to their involvement in the FPN and DMN, and their recruitment in creative cognition ${ }^{52-54}$.

Resting-state functional magnetic resonance imaging (rsfMRI) was used to assess RSN functional connectivity (FC), and ultrahigh field (7T) proton magnetic resonance spectroscopy (MRS) was used to assess glutamate concentrations in designated brain areas. Overall it was hypothesized that psilocybin would have an acute and persisting increase on outcome measures of DT, which would be predicted by within-network FC of the DMN. It was further hypothesized that psilocybin would decrease CT acutely ${ }^{20}$, which would be predicted by alterations in between-network FC of the DMN and FPN.

\section{Materials and methods}

A detailed description of the experimental procedure is provided in the Supplementary Methods and briefly summarized here.

The present study was conducted between July 2017 and June 2018 at Maastricht University, employing a balanced randomized (1:1), placebo-controlled, double-blind, parallel-group design. Sixty healthy participants, with previous experience with a psychedelic drug but not within the past 3 months, were allocated to a treatment condition $(0.17 \mathrm{mg} / \mathrm{kg}$ psilocybin or placebo, p.o.). Groups were matched for age, sex, and education level. Full demographic information on the sample is available in Mason et al. ${ }^{51}$.

Participants visited the lab on three separate occasions. The first visit included a familiarization with testing day 
procedures and completion of the creativity tasks (baseline). The second visit consisted of the formal testing day, with treatment administration (psilocybin or placebo), fMRI, and completion of the creativity tasks (acute). The third testing day took place 7 days after the acute testing day, and included the final completion of the creativity tasks (follow-up).

This study was conducted according to the code of ethics on human experimentation established by the declaration of Helsinki (1964) and amended in Fortaleza (Brazil, October 2013) and in accordance with the Medical Research Involving Human Subjects Act (WMO) and was approved by the Academic Hospital and University's Medical Ethics committee. All participants were fully informed of all procedures, possible adverse reactions, legal rights and responsibilities, expected benefits, and their right for voluntary termination without consequences. The present data were part of a larger clinical trial (Netherlands Trial Register: NTR6505) of which parts have been previously published ${ }^{51}$.

\section{Creativity tasks}

Parallel (different) versions of each task were used on the baseline, acute, and follow-up testing days. All responses were scored afterward by two independent raters who were blind to the treatment condition. The interrater reliability was calculated before averaging the ratings.

\section{Picture concept task}

The PCT was administered during peak drug effects ( $\sim 20$ min post treatment), as an assessment of both convergent and DT, and in accordance with previous studies ${ }^{20-}$ ${ }^{23}$. In brief, the PCT consists of 17 stimuli, each containing between 4 and 12 color pictures shown in a matrix. Participants were given $30 \mathrm{~s}$ per stimulus to find an association between one of the pictures in each row. Specifically, they were asked first to provide the correct solution, as there is only one correct answer. The number of correct answers served as the dependent measure of CT. In order to assess DT, participants were then asked to provide as many alternative answers as possible, which are used to calculate several parameters-i.e., fluency, originality, and the ratio of both. Fluency is defined as the number of alternative associations; originality is calculated by evaluating the originality of the alternative association relative to those provided by all other participants in a session; the ratio score is then calculated by originality/fluency.

\section{Alternative uses task}

The AUT was administered in accordance with previous publications, at $\sim 130$ min post treatment, as an assessment of $\mathrm{DT}^{46}$. Participants were asked to list as many possible uses for two common household items and given 3 minutes per item to do so. Dependent outcome variables were the same as with the PCT, with an addition of the dimension "novelty". Novelty was assessed in order to separate newly generated ideas from recollection of old ideas from memory, and was done so by asking participants after idea generation to indicate if they had given any responses that were completely new to them (i.e., they had never thought of this use before, had never seen it in a movie, etc. $)^{55}$. The number of new responses were then summed up. Additional outcome measures of the AUT that have been assessed in other studies ${ }^{56}$, but were out of the scope of this study, can be found in Supplementary Results.

\section{Subjective state}

The five dimensions of altered states of consciousness (5D-ASC) scale ${ }^{49,57}$ was administered 360 min after drug administration, as a retrospective measure of drug effects. Of specific interest for this study was the subscale "insightfulness", consisting of three questions conceptually related to spontaneous creative thinking: "I felt very profound", "I had insights into connections that had previously puzzled me", and "I had very original thoughts".

\section{Image acquisition}

Participants underwent structural MRI (50 min post treatment), single-voxel proton MRS in the MPFC (65 min post) and hippocampus ( $95 \mathrm{~min}$ post), and rsfMRI (102 $\mathrm{min}$ post), during peak subjective drug effects. Images were acquired on a MAGNETOM 7T MR scanner. At rest, 258 whole-brain EPI volumes were acquired $(\mathrm{TR}=1400 \mathrm{~ms}$; $\mathrm{TE}=21 \mathrm{~ms}$; field of view $(\mathrm{FOV})=198 \mathrm{~mm}$; flip angle $=60^{\circ}$; oblique acquisition orientation; interleaved slice acquisition; 72 slices; slice thickness $=1.5 \mathrm{~mm}$; voxel size $=1.5 \times 1.5 \times$ $1.5 \mathrm{~mm}$ ). During scanning, participants were shown a black cross on a white background, and instructed to look at the cross while attempting to clear their mind, and lay as still as possible. The resting-state instructions are important to note when comparing results across studies, as different pre-scanning instructions have been found to induce distinct resting brain states during $\mathrm{fMRI}^{58}$.

Spectroscopic voxels were placed by a trained operator at the $\mathrm{mPFC}$ (voxel size $=25 \times 20 \times 17 \mathrm{~mm}^{3}$ ) and the right hippocampus (voxel size $=37 \times 15 \times 15 \mathrm{~mm}^{3}$ ). Spectra were acquired with the stimulated echo acquisition mode ${ }^{59}$ sequence ( $\mathrm{TE}=6.0 \mathrm{~ms}, \mathrm{TR}=5.0 \mathrm{~s}, 64$ averages). Outcome measures for MRS were concentration ratios of glutamate to total creatine ( $\mathrm{tCr}$, creatine + phospho-creatine).

Detailed information regarding image acquisition and MRS quantification is previously published ${ }^{51}$.

\section{Processing of imaging data}

Resting-state data were processed and analysed using the CONN toolbox 18.b (ref. ${ }^{60}$ ). All volumes were realigned, 
unwarped, segmented into gray and white matter and cerebrospinal fluid, normalized into a standard stereotactic space (Montreal Neurological Institute), and smoothed with a $6 \mathrm{~mm}$ full-width at half-maximum Gaussian kernel. Independent component analysis (ICA) was performed using group-ICA procedures implemented in the CONN toolbox, following previously described methods ${ }^{61}$. Independent components were restricted to 20 , in order to allow comparisons with established $\mathrm{RSNs}^{62}$ and previous studies on psilocybin ${ }^{38}$ and $\mathrm{LSD}^{34,35}$. Detailed information on the ICA analysis is described in Mason et al. ${ }^{51}$.

Spectroscopy data, to assess glutamate concentrations in the mPFC and the hippocampus, were analysed with LCModel version $6.3-1 \mathrm{H}$, as described in Mason et al. ${ }^{51}$.

\section{Pharmacokinetic measures}

Venous blood samples were collected at regular time points after treatment administration (80, 150, and $360 \mathrm{~min}$ ) in order to assess blood serum concentrations of psilocin, the main metabolite of psilocybin.

\section{Statistics}

\section{Sample size calculation}

This study is part of a larger trial, assessing effects of psilocybin on creativity, cognitive flexibility, brain connectivity, and glutamate concentrations. In regards to behavioral measures, our power analysis (GPower; alpha $=$ 0.05 two-tailed; power $=0.8$ ) for measures of creativity and cognitive flexibility indicated that the required sample size for detecting medium effects is $N=48$. We opted to include 60 participants in the project to ensure sufficient power to be able to detect subtler effects. In regards to power analysis for imaging data, this is quite complex, with the most common approach in the neuroimaging community is to estimate the number of subjects from previous empirical experience in the field. Previous MRS and FC studies have employed 10-21 subjects for showing significant drug effects $^{63-65}$. Based on these experiences, we expected that we would be able to demonstrate drug effects on MRS and FC in 30 participants in each drug condition.

\section{Statistical analysis}

Statistical analysis of interrater reliability, outcome variables of the creativity tasks, subjective effects, and relative glutamate concentrations were conducted in IBM SPSS Statistics 24 . To assess the interrater reliability, intra-class correlation coefficients (ICC) and their 95\% confidence intervals were calculated based on a mean-rating $(k=2)$, consistency, two-way random-effects model for relevant PCT- and AUT-dependent variables. Reliability derived from ICC values can range from poor $(<0.5)$, to moderate (0.5-0.75), good (0.75-0.9), and excellent $(>0.90)^{66}$.

Baseline-change scores of the AUT and PCT outcome measures followed normal distributions, and the variances between groups were assumed to be similar. Outcome measures entered separate mixed model ANOVAs consisting of the between-subject factor treatment (psilocybin or placebo) and the within-subject factors time (acute and follow-up), and construct (the dependent outcome variables of the PCT and AUT). In case of a treatment $x$ time $\times$ construct interaction, independent samples $t$ tests were conducted in order to assess differences between groups. Nonparametric Mann-Whitney $U$ tests were used to assess treatment group differences in ratings of the 5DASC subscale "insightfulness", and differences in relative glutamate concentrations, as these data points did not meet the assumption of normality. The alpha criterion level of significance of all aforementioned tests was set at $p=0.05$ (two-tailed).

For the assessment of within-network FC, the unthresholded ICA component images were compared between placebo and drug conditions (two sample $t$ test). Parametric statistics were used (voxel threshold $p<0.001$ uncorrected, cluster threshold $p<0.05$ cluster size, false discovery rate (FDR) corrected, two-sided).

For the assessment of between-network FC, ICA components were thresholded at $z>2$ and binarized. The mean of the time series was extracted for every resulting ROI. Time courses between all RSNs were then compared for both conditions using bivariate correlations. The resulting correlation coefficients were compared between placebo and drug conditions (two sample $t$ test). Results were corrected for multiple comparisons using FDR.

Canonical correlations ${ }^{67}$ were conducted to evaluate the association between changes in variables that showed a significant treatment effect, including (i) outcome measures of the PCT and AUT, (ii) ratings of the 5D-ASC dimension, insightfulness, (iii) within- and between-network restingstate FC, using extracted connectivity strength (beta) values, and (iv) relative glutamate concentration levels in the mPFC and hippocampus. Variables were separated into two sets; set 1 included the cognitive and subjective measures of creativity [(i) and (ii)] and set 2 included biological variables as predictors [(iii) and (iv)]. Canonical correlations were chosen as this approach assesses the relationship between two multivariate data sets, allowing investigation of variables that may have multiple causes and effects, while also reducing the potential of type 1 error ${ }^{67}$. An iterative imputation approach was performed to fill in missing data points, when applicable.

\section{Results}

Demographic variables, psilocin blood serum concentrations, subjective state, and relative glutamate concentrations

Demographic information, mean (SE) psilocin blood serum concentrations, ratings on the $5 \mathrm{D}-\mathrm{ASC}$, and 
Table 1 Mean (SE) and independent $t$ test results (psilocybin vs placebo) of all dependent outcome variables on the AUT and PCT.

\begin{tabular}{|c|c|c|c|c|c|c|c|c|c|c|}
\hline \multirow[t]{2}{*}{ Variable } & \multicolumn{5}{|l|}{ Acute } & \multicolumn{5}{|l|}{ Long term } \\
\hline & Psilocybin & Placebo & $T$ value & $P$ value & Cohen's $d$ & Psilocybin & Placebo & $T$ value & $P$ value & Cohen's $d$ \\
\hline \multicolumn{11}{|l|}{ AUT } \\
\hline Fluency & $-6.55(1.13)$ & $-1.89(1.02)$ & -3.06 & $<0.01^{*}$ & 0.80 & $-2.44(0.89)$ & $-3.62(0.96)$ & 0.89 & 0.37 & 0.23 \\
\hline Originality & $-1.62(0.97)$ & $-0.48(1.22)$ & -0.73 & 0.47 & 0.19 & $-0.14(0.83)$ & $-0.72(0.77)$ & 0.51 & 0.61 & 0.13 \\
\hline Ratio & $0.07(0.03)$ & $0.02(0.05)$ & 0.81 & 0.42 & 0.21 & $0.04(0.03)$ & $0.05(0.04)$ & -0.34 & 0.73 & 0.10 \\
\hline Novel & $-0.31(0.76)$ & $-0.21(0.49)$ & -0.11 & 0.91 & 0.03 & $2.25(0.76)$ & $0.45(0.53)$ & 1.96 & $0.05^{*}$ & 0.52 \\
\hline \multicolumn{11}{|l|}{ PCT } \\
\hline Fluency & $-2.73(1.81)$ & $4.19(1.40)$ & -3.02 & $<0.01^{*}$ & 0.84 & $4.07(1.24)$ & $5.25(1.39)$ & -0.63 & 0.53 & 0.17 \\
\hline Originality & $-0.711(1.65)$ & $4.54(1.49)$ & -2.36 & $0.02^{*}$ & 0.65 & $1.59(1.32)$ & $5.31(1.52)$ & -1.84 & 0.07 & 0.50 \\
\hline Ratio & $0.10(0.035)$ & $0.09(0.063)$ & 0.04 & 0.96 & 0.01 & $-0.04(0.03)$ & $0.06(0.06)$ & -1.53 & 0.13 & 0.42 \\
\hline Convergent & $-1.00(0.76)$ & $1.61(0.37)$ & -3.15 & $<0.01^{*}$ & 0.85 & $1.67(0.57)$ & $3.36(0.49)$ & -2.24 & $0.03^{*}$ & 0.60 \\
\hline
\end{tabular}

*Statistical significance at the $p=0.05$ level.

relative glutamate concentrations are all previously published elsewhere ${ }^{51}$ and briefly summarized here.

The psilocybin group $(n=30)$ and the placebo group ( $n$ $=30$ ) did not differ in respect to demographic variables, such as gender, age, and drug use history. Psilocin blood serum concentrations reached a peak at $80 \mathrm{~min}$ post administration $(15.61 \pm 1.66 \mathrm{ng} / \mathrm{mL})$, while participants were in the MRI scanner, and were $12.86 \pm 1.13 \mathrm{ng} / \mathrm{mL}$ during creativity task completion (150 min post). Administration of psilocybin was also associated with increased ratings on all (sub)dimensions of the 5D-ASC, including the dimension of interest for this study, "insightfulness" ( $n=60$, $U=44.5 ; p \leq 0.001, d=1.78$ ). Finally, compared to placebo, relative glutamate concentrations were significantly higher in the $\mathrm{mPFC}$ (mean $\pm \mathrm{SE}$; psilocybin: $1.23 \pm 0.02, n=24$; placebo: $1.14 \pm 0.02, n=28 ; U=200.50, p=0.01, d=0.80$ ) and significantly lower in the hippocampus (psilocybin: $0.77 \pm 0.03, n=21$; placebo: $0.88 \pm 0.03, n=25 ; U=163.50$, $p=0.03, d=0.69$ ).

\section{Creativity tasks}

The ICC for originality was 0.95 on the PCT and 0.91 on the AUT, indicating excellent reliability. The ICC for all outcome variables, including those not within the scope of this study can be found in Table S1.

\section{Picture concept test}

Psilocybin influenced performance on the PCT in a time- and construct-dependent manner, as evidenced by a significant treatment $\times$ time $\times$ construct interaction $\left(F_{3,47}\right.$ $=4.53, p=0.007$; psilocybin $n=25$, placebo $n=26)$. Compared to placebo, psilocybin acutely decreased CT $(d$ $=0.85)$, and measures of DT including fluency $(d=0.84)$, and originality $(d=0.65)$. At the 7 -day follow-up, CT was still significantly decreased when comparing psilocybin to placebo $(d=0.60$; Table 1 and Fig. 1$)$.

\section{Alternative uses test}

Psilocybin influenced performance on the AUT in a time- and construct-dependent manner, as evidenced by a significant treatment $\times$ time $\times$ construct interaction $\left(F_{3,53}\right.$ 4.50, $p=0.007$; psilocybin $n=28$, placebo $n=29$ ). Compared to placebo, psilocybin acutely decreased fluency $(d=0.80)$. Conversely, compared to placebo psilocybin significantly increased scores of novelty $(d=0.52)$ at the follow-up (Table 1 and Fig. 1).

\section{Resting-state networks}

After quality control of the imaging data, the final sample consisted of 22 participants in the psilocybin group and 26 in the placebo group. There were no significant differences between groups in regards to head motion parameters. See Supplementary Material for exclusion criteria and assessed differences between groups.

\section{Independent component analysis}

There was a good agreement between the components identified in our analysis and the templates provided by Smith, et al. ${ }^{62}$. The results of this full analysis are published elsewhere ${ }^{51}$. For the purpose of this study, we focused on the frontoparietal network $1(r=0.50)$ and 2 $(r=0.47)$ and the DMN, which was split into subcomponents (anterior: $r=0.34$ and posterior: $r=0.52$ ), as has already been observed in multiple studies ${ }^{68,69}$. The anterior SN ( $r=0.39)$ was identified via the component of Shirer et al. ${ }^{70}$ as an SN template is not identified in ref. ${ }^{62}$. 

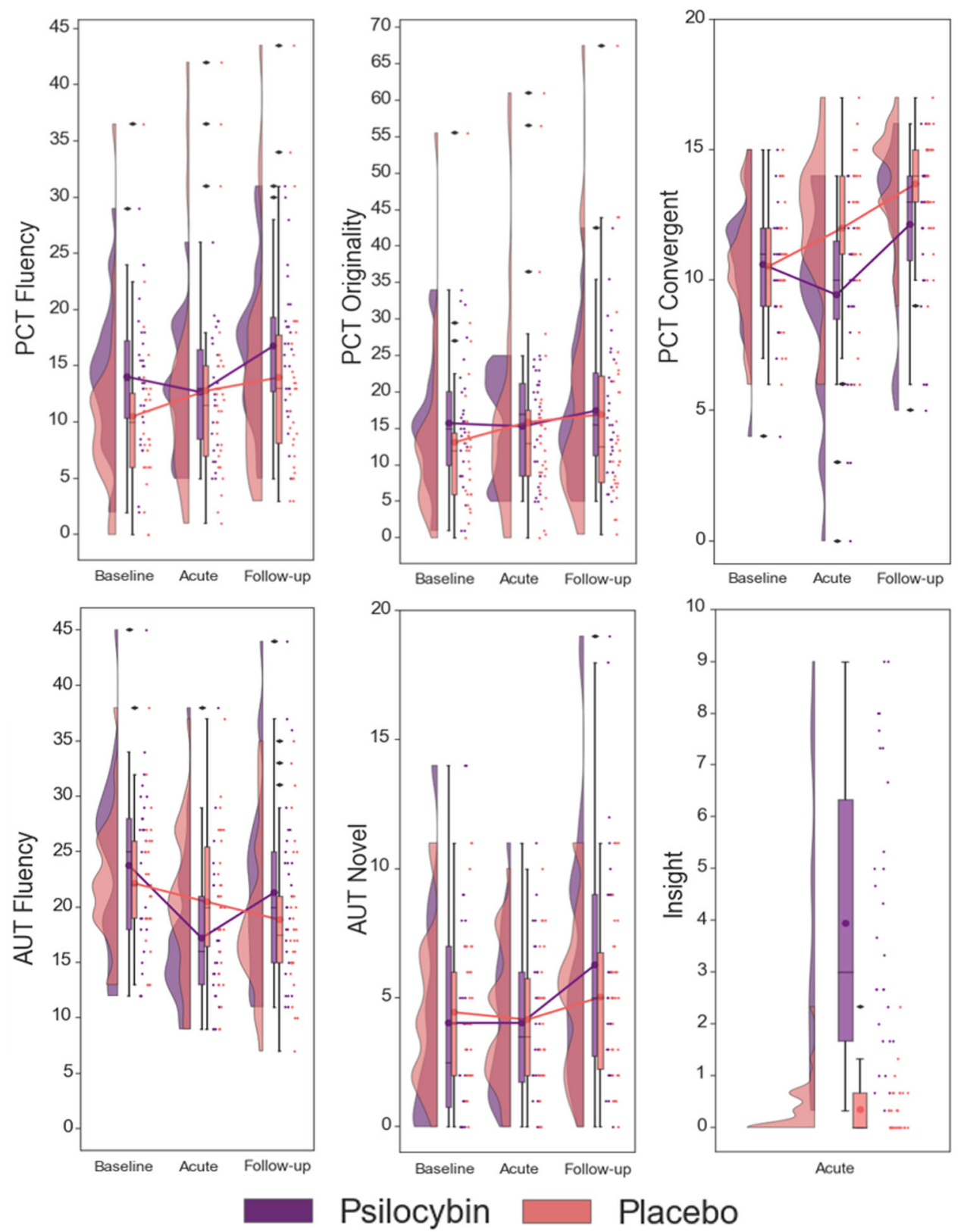

Fig. 1 Raincloud plots displaying scores on measures of creativity, which demonstrated significant differences between treatment groups. The plot consists of a probability density plot, a boxplot, and raw data points. In the boxplot, the line dividing the box represents the median of the data, the ends represent the upper/lower quartiles, and the extreme lines represent the highest and lowest values excluding outliers. The code for raincloud plot visualization has been adapted from Allen et al. ${ }^{108}$.

\section{Within-network connectivity}

Within the respective network, significantly less coactivation under the drug condition relative to placebo was found in both subcomponents of the DMN (anterior and posterior). FC within the FPN and SN did not differ between psilocybin and placebo (Fig. 2).

\section{Between-network connectivity}

Widespread increases in between-network FC were observed under psilocybin compared to placebo, with significant increases between the anterior and posterior DMN and the FPN 1, the anterior DMN and the FPN 2, and between the anterior DMN and the SN (Fig. 3). 


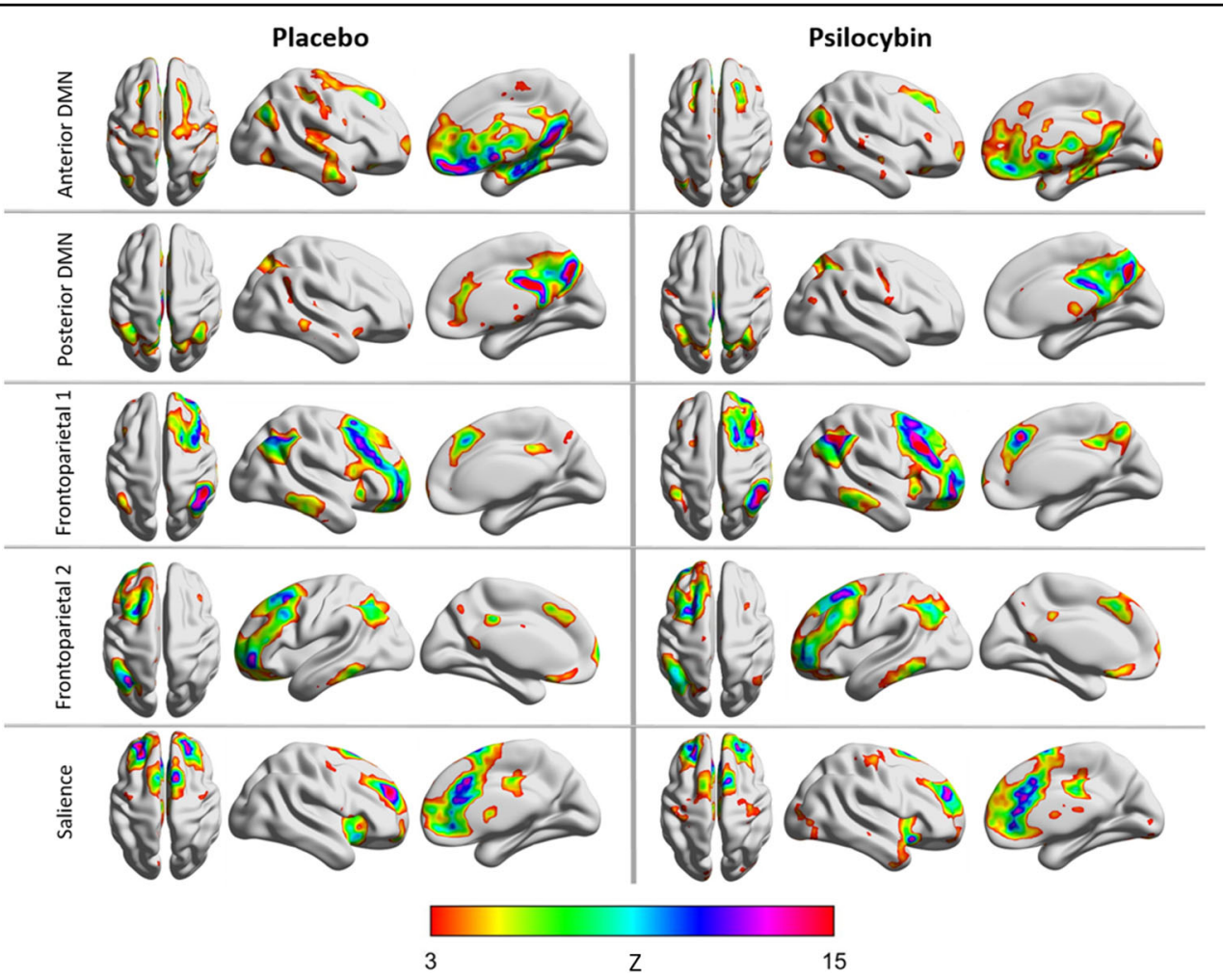

Fig. 2 Resting-state networks implicated in creative cognition, for each group (placebo and psilocybin). The anterior and posterior DMN demonstrated significant differences in within-network functional connectivity between groups.

\section{Assessing biological predictors of creativity}

A canonical correlation analysis was conducted using the eight biological variables as predictors of the eight creativity outcome measures to evaluate the multivariate shared relationship between the two variable sets. The analysis yielded seven functions with squared canonical correlations $\left(R_{\mathrm{c}}^{2}\right)$ of $0.553,0.483,0.165,0.135,0.075 .042$, and 0.001 for each successive function. The full model across all functions was statistically significant $F$ $(56,193.79)=1.48, p=0.027)$, explaining $85.3 \%$ of the variance. From this model, the first three functions were considered noteworthy in the context of this study, explaining $55.3 \%, 48.3 \%$, and $16.5 \%$ of the variance, respectively.

Table 2 presents the standardized canonical function coefficients, the structure coefficients $\left(r_{\mathrm{s}}\right)$, and the squared structure coefficients $\left(r_{\mathrm{s}}^{2}\right)$ for each function, as well as the communalities $\left(h^{2}\right)$ across the functions for each variable (for an in-depth explanation and interpretation of canonical correlations and associated terminology, the reader is referred to Sherry and Henson ${ }^{67}$ ). Function 1 indicated that the dominant contributor was ratings of insight and the secondary contributor was long-term changes in novel responses, with posterior DMN and anterior DMN FC being the dominant predictors, and secondary predictors being hippocampal glutamate levels. Overall, this function

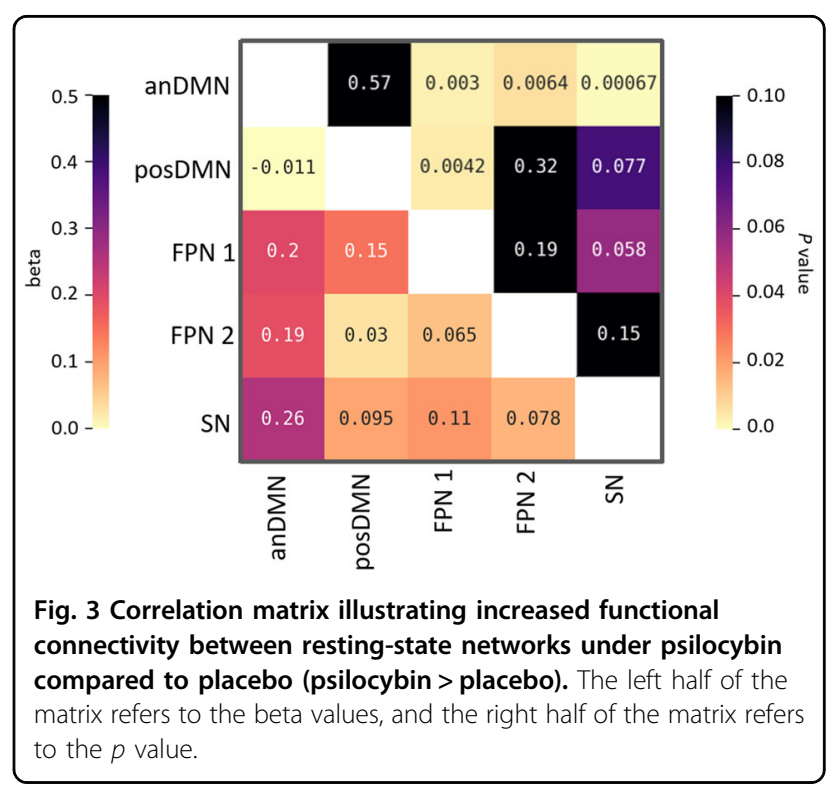

suggests that the strongest predictor of increasingly higher feelings of insight and (positive) changes in longterm novelty, were lower levels of within-network DMN FC acutely.

Function 2 indicated that the dominant contributors were the acute changes in originality, with the dominant 
Table 2 Canonical solution for biological variables predicting creativity variables for all functions.

\begin{tabular}{|c|c|c|c|c|c|c|c|c|c|c|}
\hline \multirow[b]{2}{*}{ Variable } & \multicolumn{3}{|c|}{ Function 1} & \multicolumn{3}{|c|}{ Function 2} & \multicolumn{3}{|c|}{ Function 3} & \multirow[b]{2}{*}{$h^{2}(\%)$} \\
\hline & Coef & $r_{\mathrm{s}}$ & $r_{\mathrm{s}}^{2}(\%)$ & Coef & $r_{\mathrm{s}}$ & $r_{s}^{2}(\%)$ & Coef & $r_{\mathrm{s}}$ & $r_{\mathrm{s}}^{2}(\%)$ & \\
\hline AUT fluency & 0.10 & -0.40 & 15.68 & -0.14 & -0.04 & 0.12 & 0.08 & -0.21 & 4.54 & 20.34 \\
\hline PCT fluency & -0.02 & -0.41 & 16.73 & 1.45 & -0.02 & 0.06 & 0.44 & 0.32 & 10.24 & 27.03 \\
\hline PCT originality & -0.17 & -0.45 & 20.70 & -1.66 & $-\underline{0.49}$ & 23.72 & -0.33 & 0.22 & 4.80 & $\underline{49.22}$ \\
\hline PCT convergent & 0.11 & -0.38 & 14.67 & 0.11 & 0.08 & 0.61 & 0.75 & $\underline{0.78}$ & 61.00 & 76.27 \\
\hline Insight & 0.83 & $\underline{0.89}$ & 79.21 & -0.39 & -0.21 & 4.37 & 0.62 & 0.29 & 8.12 & $\underline{91.70}$ \\
\hline PCT convergent long term & -0.32 & -0.44 & 19.71 & -0.02 & 0.03 & 0.10 & 0.22 & $\underline{0.58}$ & 33.52 & $\underline{53.33}$ \\
\hline AUT novelty long term & 0.24 & $\underline{0.47}$ & 22.37 & 0.33 & 0.38 & 14.75 & -0.21 & -0.21 & 4.33 & 41.44 \\
\hline$R_{c}^{2}$ & & & 55.3 & & & 48.3 & & & 16.5 & \\
\hline AnDMN & -0.67 & $-\underline{0.80}$ & 63.36 & -0.94 & -0.17 & 3.03 & -0.18 & 0.04 & 0.17 & $\underline{66.56}$ \\
\hline PosDMN & -0.30 & $-\underline{0.72}$ & 52.13 & 0.92 & $\underline{0.56}$ & 30.80 & -0.38 & -0.15 & 2.22 & $\underline{85.15}$ \\
\hline anDMN-SN & 0.10 & 0.34 & 11.70 & 0.13 & -0.20 & 3.88 & -0.38 & $-\underline{0.53}$ & 28.20 & 43.77 \\
\hline anDMN-FPN 1 & 0.25 & 0.28 & 7.90 & -0.39 & -0.36 & 12.74 & -0.40 & $-\underline{0.67}$ & 44.76 & $\underline{65.40}$ \\
\hline posDMN-FPN 1 & 0.07 & 0.24 & 5.86 & 0.12 & 0.15 & 2.16 & -0.42 & $-\underline{0.70}$ & 49.14 & $\underline{57.16}$ \\
\hline anDMN-FPN 2 & -0.53 & 0.11 & 1.10 & -0.42 & -0.35 & 12.53 & -0.14 & $-\underline{0.46}$ & 21.44 & 35.07 \\
\hline Hippocampal glutamate & -0.22 & $-\underline{0.49}$ & 24.30 & 0.18 & 0.21 & 4.28 & -0.10 & -0.30 & 8.94 & 37.53 \\
\hline mPFC glutamate & 0.21 & 0.37 & 13.62 & 0.24 & 0.03 & 0.07 & -0.28 & -0.30 & 9.18 & 22.87 \\
\hline
\end{tabular}

$r_{\mathrm{s}}>|0.45|$ and $h^{2}>45 \%$ are underlined and deemed valuable contributors.

predictor being posterior DMN FC, suggesting that the strongest predictor of larger (negative) changes in acute originality were lower levels of within-network posterior DMN FC.

Function 3 indicated that the dominant contributors were the acute and long-term changes in $\mathrm{CT}$, whereas the dominant predictors were FC between both components of the DMN and the FPN. This function suggests that the strongest predictor of larger (negative) changes in acute and long-term CT were higher levels of between-network FC between the DMN and the FPN.

\section{Discussion}

The present study demonstrates the first modern-day attempt to experimentally assess the (sub)acute effects of a psychedelic on divergent and $\mathrm{CT}$, the two constructs of creative thinking. Findings demonstrate that psilocybin induces a time- and construct-related differentiation of effects on creative thinking; whereas CT and aspects of DT were shown to decrease during the psychedelic state, an aspect of DT was shown to increase 7 days later compared to placebo. Acutely, individuals under psilocybin also reported higher subjective ratings on a measure akin to spontaneous creative thinking. Analyses indicated that alterations in creative thinking were further predicted by acute alterations in RSFC. Whereas disintegration of the DMN predicted both higher scores in spontaneous creative thinking and long-term increases in novelty of generated ideas, increased connectivity between the DMN and the FPN predicted decreases in acute and longterm CT.

\section{Acute and persisting effects of psilocybin on divergent thinking}

Contrary to our hypothesis, results indicate that aspects of DT, or the idea generation phase of the creative process, are impaired during the acute psychedelic state. Namely, participants generated less ideas and associations when under psilocybin compared to placebo, as indicated by significantly lower fluency scores on the AUT and the PCT, respectively. Originality scores were also lower after psilocybin on the PCT; however, when controlling quality for quantity (how many responses were given; ratio), there were no significant differences. Thus, when assessing how unique generated responses were, there was no significant difference between treatments. That said, individuals under the influence of psilocybin reported significantly higher ratings of "insightfulness", a construct conceptually related to spontaneous creative thinking (e.g., "I had insights into connections that had previously puzzled me" and "I had very original thoughts"). Thus, overall, results suggest psilocybin acutely reduces an individual's volume 
of new ideas and associations, without modulating quality, in response to goal-directed creativity tasks, while increasing feelings of spontaneous creative insights.

In line with our hypothesis, results indicate an increase in aspects of DT after the acute drug effects have worn off. Namely, 7 days after psilocybin administration, participants generated a higher quantity of novel ideas for uses of an everyday object on the AUT compared to placebo. That said, there were no differences in amount of generated ideas or associations in total, or how original responses were. It should also be noted that the effect size on number of novel ideas was moderate $(d=0.52)$, however, is in accordance with other studies that have found persisting changes in aspects related to DT at various time points relative to consumption of a range of psychedelic drugs (see Aday et al. ${ }^{71}$ for a review).

\section{Acute and persisting effects of psilocybin on convergent thinking}

CT can be seen as the second phase in the creative process, focused on evaluating possibilities generated during DT, and narrowing them down to a logical solution ${ }^{4}$. In line with previous studies suggesting that psychedelics decrease conventional, logical thinking ${ }^{16,17,20}$, we found an acute decrement in CT when comparing psilocybin to placebo. When comparing the two treatment groups, we also found that the psilocybin group performed significantly worse compared to placebo at follow-up. However, it should be noted that although there is a significant difference when looking between groups, when looking within groups, there is an increase in performance on the CT in the placebo group when comparing baseline to acute and follow-up, and an increase in performance in the psilocybin group when comparing baseline to the follow-up. Thus, although measures were taken to reduce potential learning effects on the PCT, including administering three different versions of the task, and withholding feedback on performance, our data indicate that this could be taking place. The finding that psilocybin acutely blocked this learning effect adds to the literature that psychedelics disrupt associative learning ${ }^{72}$ and impair executive functioning ${ }^{73}$; effects that have been proposed to be mediated via agonistic activation at the $5-\mathrm{HT}_{2 \mathrm{~A}}$ receptor $^{73}$, and potentially due to impaired attentional performance resulting from an inability to ignore taskirrelevant stimuli ${ }^{74,75}$. This suggestion of learning effects also brings into question findings from recent naturalistic studies that found persisting enhancements in CT on the PCT after intake of a psychedelic ${ }^{21-23,76}$, and emphasizes the importance of a placebo control.

\section{Do psychedelics enhance creative thinking?}

Results of our study suggests that psychedelics do not enhance creative thinking per se, but rather mediate changes in particular constructs of creative thinking, in a time-dependent manner. Interestingly, these constructs could be interpreted in two different ways, either emphasizing a discrepancy between objective vs subjective creative performance, or highlighting the difference between spontaneous vs deliberate creative cognition.

In regards to the former, our data could suggest that psilocybin acutely impairs the idea generation and evaluation phase of creative thinking, while enhancing the feelings of quality of generated ideas (measured via the subjective questionnaire of insightfulness), despite lack of objective evidence. This discrepancy between a subjective sense of enhanced creative thinking, and objective assessments of creativity is a mismatch that is also found in the early psychedelic creativity literature ${ }^{10}$. Increased feelings of insight, changes in affect, and attribution of meaning to previously neutral stimuli are recognized as common acute effects of psychedelic drugs ${ }^{77-82}$, and thus it is plausible that reports of increased creativity during the acute psychedelic state are due to a subjective sense of creativity enhancement that does not match the objective quality ${ }^{1}$. However, 1 week later this discrepancy appeared to dissipate, in that it was objectively found that the idea generation phase is enhanced, with a higher number of novel ideas reported after psilocybin.

An alternative interpretation of our data could arise from the difference between two processing modes of creative cognition, deliberate vs spontaneous ${ }^{48}$. The deliberate processing mode is initiated when one is focused on meeting explicit task demands, such as in a guided, timed situation (as is the case with our tasks), and is characterized as being more attention-demanding, searching along structured and rational belief systems in order to reach a goal ${ }^{48}$. In contrast, spontaneous insights tend to occur in a mental state characterized by unrestrained cognition and defocused attention, and tend to be more random, unfiltered, and bizarre ${ }^{48}$; a mental state almost synonymous with the characterization of the psychedelic state ${ }^{83}$. Thus, this discrepancy between acute increases in feelings of insight, but decreased number of ideas, could suggest that psychedelics acutely increase the potential for spontaneous creative thought, while decreasing the potential for deliberate creative cognition. As it has been suggested that productive creative cognition may likely be facilitated by a balance between spontaneous insights and controlled deliberate processing ${ }^{47}$, our data suggest that psychedelics disrupt this balance acutely, whereas subacutely this balance is restored, with deliberate creative cognition potentially even enhanced, as evidenced by an increased number of novel ideas one week later. A post hoc correlation analysis also indicates that there may be a relationship between acute increases in spontaneous creativity, and long-term increases in idea 
generation, as a significant positive correlation was found between the two (see Supplemenatary Results).

\section{Biological predictors of creative thinking}

As we found that psychedelics can mediate changes in particular constructs of creative thinking, it suggests that they could be a novel tool to investigate underlying neural mechanisms of the creative process. Thus, canonical correlations were conducted to predict performance on DT and CT from acute biological parameters hypothesized to underlie creative cognition, including within- and between-network FC of the DMN, FPN, and SN, and glutamate concentrations in the $\mathrm{mPFC}$ and the hippocampus.

Decreased integrity of the DMN was found to be the strongest predictor in regards to increased feelings of acute subjective spontaneous creative thinking (insightfulness). In general, this is in line with previous work that has implicated the DMN in subjective effects representing unconstrained cognition during the psychedelic state $^{1,43,81,84}$, as well as the spontaneous processing mode in creative cognition ${ }^{47}$. Changes in DMN activity and integrity have been repeatedly found during the acute psychedelic state ${ }^{33,34,36,81,85}$, and a disruption of coupling between the medial temporal lobes (MTLs) and the $\mathrm{DMN}^{29}$, has been particularly implicated in explaining some of the key subjective effects of the drugs, including feelings of insightfulness ${ }^{81}$, and an altered sense of self and reality ${ }^{84,86}$. In addition, DMN and MTL activity have both been implicated in dreaming ${ }^{87}$, a state neurophenomenologically similar to the psychedelic state $e^{1,83,88,89}$ in that both are characterized by experience of bizarre cognitive phenomena, illogical transitions between thoughts, and vivid sensorimotor imagery ${ }^{90}$. Interestingly, dreaming has been suggested to be the most extreme form of the spontaneous processing mode in creative cognition ${ }^{48}$. Thus taken together, our data suggest that the psychedelic state may increase the potential for spontaneous creative thought, and does so via disruption of DMN integrity. In addition, we found that decreased glutamate in the hippocampus was a secondary predictor in increased ratings of insight. As it has been suggested that MTL activity is a crucial driver of the $\mathrm{DMN}^{29}$, and the relationship between the two has been implicated in the psychedelic and related states, it could be suggested that glutamatergic activity of hippocampal regions could underlie this desynchronization. The involvement of the hippocampus in creative cognition is also interesting to speculate on, as it has been found that past knowledge about a problem can actually be detrimental to solving it, constraining potential ideas to what is already known, and thus is suggested to be a disadvantage of the deliberate mode of creative cognition, and an advantage of the spontaneous mode(see Dietrich ${ }^{48}$ for a review).
In regards to deliberate, task-based creative thinking, the finding that decreased within-network DMN FC predicted both an acute decrease in scores of originality, and a long-term increase in generation of novel ideas may seem counterintuitive, however, actually is in line with previous work. Specifically, given the suggested role of the DMN in underlying the idea generation process of DT, an acute decrement in DMN FC resulting in an acute decrement in DT performance on a creativity task would be expected. In addition, previous work has found that while psychedelics decrease within-network DMN FC acutely, they increase DMN integrity subacutely ${ }^{41-44}$, potentially via a neuroplastic effect on brain network function $^{44,91,92}$. Thus, it could be suggested that the latter prediction seen here is reflecting the subsequent psilocybin-induced increase in DMN FC that could facilitate an increase in novel ideas. Such findings add support to the suggestion that novel idea generation is mediated via DMN network $\mathrm{FC}^{1,25-27}$, and also suggest that current frameworks conceptualizing the impact of the psychedelic state on creativity could be adapted to include both acute and persisting effects of the drug ${ }^{1}$.

Finally, levels of acute connectivity between both subcomponents of the DMN and the FPN were found to inversely predict acute and long-term changes in CT performance. Namely, increasingly positive DMN-FPN connectivity predicted increasingly worse CT performance relative to baseline. Although usually anticorrelated $^{93,94}$, previous studies have found that, during a task, higher dynamic DMN-FPN connectivity predicted better performance in areas, such as creative cognition, cognitive flexibility, attention, and learning ${ }^{25,26,95-98}$. In regards to creative cognition in particular, it has been suggested that during the creative process, the degree of FPN involvement increases as the need for more deliberate constraints on thought content (in order to reach, e.g., a goal) increases ${ }^{1}$. Further, it has been suggested that productive creative cognition is facilitated by a balance between spontaneous and controlled processing ${ }^{48}$, both of which may be experienced concurrently via coupling between the DMN and task positive networks like the $\mathrm{FPN}^{47}$. Thus, the association between DMN-FPN connectivity and $\mathrm{CT}$ is in line with previous work implicating DMN-FPN dynamics in the idea evaluation phase of the creative process. That said, it may then be hypothesized that increased DMN-FPN coupling would result in better performance on a task of CT. However, the aforementioned evidence stems from measurements taken while individuals were performing a task. Instead, it has been found that higher resting-state FPN-DMN dynamics relates to poorer performance on tasks of cognitive flexibility, and has also been found in states characterized by poorer attention, including during the psychedelic state ${ }^{38}$, and in patients with ADHD ${ }^{99}$. Thus increased DMN-FPN 
between-network connectivity at rest could be reflecting the potential of either intrusion of, or insufficient suppression of, the DMN during a task. As increased restingstate DMN-FPN connectivity has also been found in patients characterized by poorer attentional abilities, and psychedelics have been found to disrupt executive functions potentially via impairment of attention ${ }^{74,75}$, this could provide a biological basis for how psychedelics impair executive functioning, and could explain how psilocybin blocked the suggested CT learning effect seen during our study.

Although the role of the mPFC has been strongly highlighted in creative cognition ${ }^{48}$, glutamate concentrations in this area did not play a significant role in the canonical model. This could suggest that the involvement of the $\mathrm{mPFC}$ is due to its role in integration and interaction of distributed brain areas operating in large-scale networks ${ }^{100}$, rather than underlying neural activity in the specific brain area. In addition, although the connectivity between the DMN and the $\mathrm{SN}$ has been implicated in the creative process, in that the $\mathrm{SN}$ has been suggested to facilitate the shift of ideas from the DMN to the $\mathrm{FPN}^{25-27}$, we did not find that this strongly predicted any outcome measures of creative thinking. The latter may be due to the fact that we are correlating network activity at rest, with behavior measures taken outside of the scanner, emphasizing a limitation of this study. Thus, although consistent with the previously discussed literature, it is important to note that discrepancies have been found between state and trait network connectivity and creative performance ${ }^{101,102}$. In light of this, results should be taken as preliminary, and future studies assessing the neurobiological effects of psychedelics on creativity should employ creativity tasks during scanning.

\section{Conclusion}

In conclusion, this study found that psilocybin induces a time- and construct-related differentiation of effects on creative thinking, suggesting that psychedelics could be a novel tool to investigate underlying neural mechanisms of the creative process ${ }^{1,24}$. In addition, these findings add some support to the historical claims that psychedelics can influence aspects of the creative process, reducing conventional, logical thinking, and giving rise to novel thoughts, but emphasizes the distinction between spontaneous and deliberate creative cognition, as well as acute and persisting effects of the drug.

These distinctions are of particular importance, as psychedelics are currently being investigated to treat a number of mental health disorders ${ }^{103}$, characterized by rigid, inflexible thought patterns ${ }^{29,104,105}$. Thus, it could be suggested that the ability of psilocybin to acutely decrease CT and increase spontaneous DT, while subacutely enhancing more goal-directed DT, could aid in the therapeutic process by opening up a window of opportunity where therapeutic interventions could prove more effective. Namely, while under the influence of a psychedelic, rigid thought content (CT) could be decreased, while unguided, spontaneous thoughts may give rise to new insights and perspectives of previous events and current problems ${ }^{19,106,107}$. Subacutely, patients may then be able to integrate these insights with a therapist, and come up with new, more effective strategies that facilitate adaptive interpretation and coping abilities ${ }^{8}$. Nevertheless, future studies should be employed in clinical populations in order to assess this proposal, as well as the underlying neurobiological mechanisms.

\begin{abstract}
Acknowledgements
The authors would like to thank Cees van Leeuwen for medical supervision, Fulya Onus and Sofia Rocha for their assistance with data collection, Safak Erener for assistance with rating responses, Pablo Mallaroni for his comments on the manuscript, and all participants for their time and effort. This study is part of the Beckley/Maastricht Research Programme. The Beckley Foundation made a financial contribution to the study.
\end{abstract}

\section{Author details}

'Department of Neuropsychology and Psychopharmacology, Faculty of Psychology and Neuroscience, Maastricht University, P.O. Box 616, 6200 MD Maastricht, the Netherlands. ${ }^{2}$ Department of Psychiatry (UPK), University of Basel, Basel, Switzerland. 'Institute of Legal Medicine, University of Frankfurt, Kennedyallee 104, D-60596 Frankfurt/Main, Germany. ${ }^{4}$ The Beckley Foundation, Beckley Park, Oxford OX3 9SY, UK

\section{Author contributions}

N.M., K.K., J.G., and A.F. designed the research. N.M. and J.R. performed the research. D.T. set up the MR/MRS sequences. N.M., F.M., and B.D.R. analyzed the data. All authors made a substantial contribution to interpretation of the data and drafting of the manuscript.

Conflict of interest

The authors declare no competing interests.

\section{Publisher's note}

Springer Nature remains neutral with regard to jurisdictional claims in published maps and institutional affiliations.

Supplementary information The online version contains supplementary material available at https://doi.org/10.1038/s41398-021-01335-5.

Received: 25 September 2020 Revised: 18 March 2021 Accepted: 29 March 2021

Published online: 08 April 2021

\footnotetext{
References

1. Girn, M., Mills, C., Roseman, L., Carhart-Harris, R. L. \& Christoff, K. Updating the dynamic framework of thought: Creativity and psychedelics. Neurolmage 213, 116726 (2020). 2020.116726.

2. Runco, M. A. in Dynamic Perspectives on Creativity, 181-188 (Springer, 2019).

3. Guilford, J. P. The Nature of Human Intelligence (McGraw-Hill, 1967).

4. Runco, M. A. \& Jaeger, G. J. The standard definition of creativity. Creativity Res. J. 24, $92-96$ (2012).

5. Cropley, A. In praise of convergent thinking. Creativity Res. J. 18, 391-404 (2006).

6. Fresco, D. M., Williams, N. L. \& Nugent, N. R. Flexibility and negative affect: examining the associations of explanatory flexibility and coping flexibility to each other and to depression and anxiety. Cogn. Ther. Res. 30, 201-210 (2006).
} 
7. Forgeard, M. J. C. \& Elstein, J. G. Advancing the clinical science of creativity. Front. Psychol. 5, 613 (2014).

8. Forgeard, M. J. C. \& Eichner, K. V. in The Wiley Blackwell Handbook of Positive Psychological Interventions, 137-154 (Wiley-Blackwell, 2014).

9. Nichols, D. E. Psychedelics. Pharmacol. Rev. 68, 264-355 (2016).

10. Baggott, M. J. Psychedelics and creativity: a review of the quantitative literature. PeerJ Prepr. 3, e1202v1201, (2015).

11. Sessa, B. Is it time to revisit the role of psychedelic drugs in enhancing human creativity? J. Psychopharmacol. 22, 821-827 (2008).

12. McGlothlin, W., Cohen, S. \& McGlothlin, M. S. Long lasting effects of LSD on normals. Arch. Gen. Psychiatry 17, 521-532 (1967).

13. Zegans, L. S., Pollard, J. C. \& Brown, D. The effects of LSD-25 on creativity and tolerance to regression. Arch. Gen. Psychiatry 16, 740-749 (1967).

14. Harman, W. W., McKim, R. H., Mogar, R. E., Fadiman, J. \& Stolaroff, M. J. Psychedelic agents in creative problem-solving: a pilot study. Psychol. Rep. 19, 211-227 (1966).

15. Preller, $\mathrm{K} . \mathrm{H}$. et al. The fabric of meaning and subjective effects in LSDinduced states depend on serotonin 2A receptor activation. Curr. Biol. 27, 451-457 (2017).

16. Kraehenmann, R. et al. LSD increases primary process thinking via serotonin 2A receptor activation. Front. Pharmacol. 8, 814 (2017).

17. Family, N. et al. Semantic activation in LSD: evidence from picture naming. Lang. Cogn. Neurosci. 31, 1320-1327 (2016).

18. Murphy-Beiner, A. \& Soar, K. Ayahuasca's 'afterglow': improved mindfulness and cognitive flexibility in ayahuasca drinkers. Psychopharmacology 237, 1161-1169 (2020).

19. Frecska, E., Móré, C. E., Vargha, A. \& Luna, L. E. Enhancement of creative expression and entoptic phenomena as after-effects of repeated ayahuasca ceremonies. J. Psychoact. Drugs 44, 191-199 (2012).

20. Kuypers, K. P. C. et al. Ayahuasca enhances creative divergent thinking while decreasing conventional convergent thinking. Psychopharmacology 233, 3395-3403 (2016).

21. Mason, N. L., Mischler, E., Uthaug, M. V. \& Kuypers, K. P. Sub-acute effects of psilocybin on empathy, creative thinking, and subjective well-being. J. Psychoact. Drugs 51, 123-134 (2019).

22. Uthaug, M. V. et al. A single inhalation of vapor from dried toad secretion containing 5-methoxy-N,N-dimethyltryptamine (5-MeO-DMT) in a naturalistic setting is related to sustained enhancement of satisfaction with life, mindfulness-related capacities, and a decrement of psychopathological symptoms. Psychopharmacology 236, 2653-2666 (2019).

23. Uthaug, M. V. et al. Sub-acute and long-terrom effects of ayahuasca on affect and cognitive thinking style and their association with ego dissolution. Psychopharmacology 235, 1-11 (2018).

24. Kuypers, K. P. C. Out of the box: a psychedelic model to study the creative mind. Med. Hypotheses 115, 13-16 (2018).

25. Beaty, R. E., Benedek, M., Barry Kaufman, S. \& Silvia, P. J. Default and executive network coupling supports creative idea production. Sci. Rep. $\mathbf{5}$, 10964 (2015).

26. Beaty, R. E. et al. Robust prediction of individual creative ability from brain functional connectivity. Proc. Natl Acad. Sci. USA 115, 1087-1092 (2018).

27. Beaty, R. E., Benedek, M., Silvia, P. J. \& Schacter, D. L. Creative cognition and brain network dynamics. Trends Cogn. Sci. 20, 87-95 (2016).

28. Christoff, K., Ivving, Z. C., Fox, K. C. R., Spreng, R. N. \& Andrews-Hanna, J. R. Mind-wandering as spontaneous thought: a dynamic framework. Nat. Rev. Neurosci. 17, 718-731 (2016).

29. Carhart-Harris, R. L. et al. The entropic brain: a theory of conscious states informed by neuroimaging research with psychedelic drugs. Front. Hum. Neurosci. 8 (2014).

30. Carhart-Harris, R. L. \& Friston, K. J. REBUS and the anarchic brain: toward a unified model of the brain action of psychedelics. Pharmacol. Rev. 71, 316-344 (2019).

31. Carhart-Harris, R. L. et al. The paradoxical psychological effects of lysergic acid diethylamide (LSD). Psychol. Med. 46, 1379-1390 (2016).

32. Muthukumaraswamy, S. D. et al. Broadband cortical desynchronization underlies the human psychedelic state. J. Neurosci. 33, 15171-15183 (2013).

33. Palhano-Fontes, F. et al. The psychedelic state induced by ayahuasca modulates the activity and connectivity of the default mode network. PLOS ONE 10, e0118143 (2015).

34. Müller, F., Dolder, P. C., Schmidt, A., Liechti, M. E. \& Borgwardt, S. Altered network hub connectivity after acute LSD administration. Neurolmage Clin. 18, 694-701 (2018).
35. Carhart-Harris, R. L. et al. Neural correlates of the LSD experience revealed by multimodal neuroimaging. Proc. Natl Acad. Sci. USA 113, 4853-4858 (2016).

36. Carhart-Harris, R. L. et al. Neural correlates of the psychedelic state as determined by fMRI studies with psilocybin. Proc. Natl Acad. Sci. USA 109, 2138-2143 (2012).

37. Tagliazucchi, E., Carhart-Harris, R., Leech, R., Nutt, D. \& Chialvo, D. R. Enhanced repertoire of brain dynamical states during the psychedelic experience. Hum. Brain Mapp. 35, 5442-5456 (2014).

38. Carhart-Harris, R. L. et al. Functional connectivity measures after psilocybin inform a novel hypothesis of early psychosis. Schizophr. Bull. 39, 1343-1351 (2013).

39. Roseman, L., Leech, R., Feilding, A., Nutt, D. J. \& Carhart-Harris, R. L. The effects of psilocybin and MDMA on between-network resting state functional connectivity in healthy volunteers. Front. Hum. Neurosci. 8 (2014).

40. Tagliazucchi, E. et al. Increased global functional connectivity correlates with LSD-induced ego dissolution. Curr. Biol. 26, 1043-1050 (2016).

41. Sampedro, F. et al. Assessing the psychedelic "after-glow" in ayahuasca users: post-acute neurometabolic and functional connectivity changes are associated with enhanced mindfulness capacities. Int. J. Neuropsychopharmacol. 20, 698-711 (2017).

42. Carhart-Harris, R. L. et al. Psilocybin for treatment-resistant depression: fMRLmeasured brain mechanisms. Sci. Rep. 7, 13187 (2017).

43. Smigielski, L., Scheidegger, M., Kometer, M. \& Vollenweider, F. X. Psilocybinassisted mindfulness training modulates self-consciousness and brain default mode network connectivity with lasting effects. Neurolmage 196, 207-215 (2019).

44. Barrett, F. S., Doss, M. K., Sepeda, N. D., Pekar, J. J. \& Griffiths, R. R. Emotions and brain function are altered up to one month after a single high dose of psilocybin. Sci. Rep. 10, 2214 (2020).

45. Pasquini, L., Palhano-Fontes, F. \& Araujo, D. B. Subacute effects of the psychedelic ayahuasca on the salience and default mode networks. J. Psychopharmacol 34, 623-635, https://doi.org/10.1177/0269881120909409 (2020).

46. Kaufman, J. C., Plucker, J. A. \& Baer, J. Essentials of Creativity Assessment, Vol. 53 (Wiley, 2008).

47. Mok, L. W. The interplay between spontaneous and controlled processing in creative cognition. Front Hum. Neurosci. 8, 663 (2014).

48. Dietrich, A. The cognitive neuroscience of creativity. Psychon. Bull. Rev. 11, 1011-1026 (2004).

49. Dittrich, A. The standardized psychometric assessment of altered states of consciousness (ASCS) in humans. Pharmacopsychiatry 31, 80-84 (1998).

50. Aghajanian, G. K. \& Marek, G. J. Serotonin model of schizophrenia: emerging role of glutamate mechanisms. Brain Res. Brain Res. Rev. 31, 302-312 (2000).

51. Mason, N. L. et al. Me, myself, bye: regional alterations in glutamate and the experience of ego dissolution with psilocybin. Neuropsychopharmacology $\mathbf{4 5}$, 2003-2011, https://doi.org/10.1038/s41386-020-0718-8 (2020).

52. Fink, A. et al. Enhancing creativity by means of cognitive stimulation: evidence from an fMRI study. Neurolmage 52, 1687-1695 (2010).

53. Beaty, R. E. et al. Default network contributions to episodic and semantic processing during divergent creative thinking: a representational similarity analysis. Neurolmage 209, 116499 (2020).

54. Madore, K. P., Thakral, P. P., Beaty, R. E., Addis, D. R. \& Schacter, D. L. Neural mechanisms of episodic retrieval support divergent creative thinking. Cereb. Cortex 29, 150-166 (2017).

55. Benedek, M. et al. To create or to recall? Neural mechanisms underlying the generation of creative new ideas. Neurolmage 88, 125-133 (2014).

56. Silvia, P. J. et al. Assessing creativity with divergent thinking tasks: exploring the reliability and validity of new subjective scoring methods. Psychol. Aesthet. Creat. Arts 2, 68 (2008).

57. Studerus, E., Gamma, A. \& Vollenweider, F. X. Psychometric evaluation of the altered states of consciousness rating scale (OAV). PLOS ONE $\mathbf{5}$, e12412 (2010).

58. Kawagoe, T., Onoda, K. \& Yamaguchi, S. Different pre-scanning instructions induce distinct psychological and resting brain states during functional magnetic resonance imaging. Eur. J. Neurosci. 47, 77-82 (2018).

59. Frahm, J. et al. Localized high-resolution proton NMR spectroscopy using stimulated echoes: initial applications to human brain in vivo. Magn. Reson. Med. 9, 79-93 (1989).

60. Whitfield-Gabrieli, S. \& Nieto-Castanon, A. Conn: a functional connectivity toolbox for correlated and anticorrelated brain networks. Brain Connect. 2 125-141 (2012). 
61. Calhoun, V. D., Adali, T., Pearlson, G. D. \& Pekar, J. J. A method for making group inferences from functional MRI data using independent component analysis. Hum. Brain Mapp. 14, 140-151 (2001).

62. Smith, S. M. et al. Correspondence of the brain's functional architecture during activation and rest. Proc. Natl Acad. Sci. USA 106, 13040-13045 (2009).

63. Mason, N. L. et al. Cannabis induced increase in striatal glutamate associated with loss of functional corticostriatal connectivity. Eur. Neuropsychopharmacol. 29, 247-256 (2018)

64. Preller, K. H. et al. Effects of serotonin 2A/1A receptor stimulation on social exclusion processing. Proc. Natl Acad. Sci. USA 113, 5119-5124 (2016).

65. Mason, N. L. et al. Reduced responsiveness of the reward system is associated with tolerance to cannabis impairment in chronic users. Addict. Biol. 26 e12870 (2021).

66. Koo, T. K. \& Li, M. Y. A guideline of selecting and reporting intraclass correlation coefficients for reliability research. J. Chiropr. Med. 15, 155-163 (2016).

67. Sherry, A. \& Henson, R. K. Conducting and interpreting canonical correlation analysis in personality research: A user-friendly primer. J. Personal. Assess. 84, 37-48 (2005).

68. Abou-Elseoud, A. et al. The effect of model order selection in group PICA. Hum. Brain Mapp. 31, 1207-1216 (2010).

69. Allen, E. A. et al. A baseline for the multivariate comparison of resting-state networks. Front. Syst. Neurosci. 5, 2-2 (2011).

70. Shirer, W. R., Ryali, S., Rykhlevskaia, E., Menon, V. \& Greicius, M. D. Decoding subject-driven cognitive states with whole-brain connectivity patterns. Cereb. Cortex 22, 158-165 (2012).

71. Aday, J. S., Mitzkovitz, C. M., Bloesch, E. K., Davoli, C. C. \& Davis, A. K. Long-term effects of psychedelic drugs: a systematic review. Neurosci. Biobehav. Rev. 113 179-189 (2020).

72. Barrett, F. S., Carbonaro, T. M., Hurwitz, E., Johnson, M. W. \& Griffiths, R. R. Double-blind comparison of the two hallucinogens psilocybin and dextromethorphan: effects on cognition. Psychopharmacology 235, 2915-2927 (2018).

73. Pokorny, T., Duerler, P., Seifritz, E., Vollenweider, F. X. \& Preller, K. H. LSD acutely impairs working memory, executive functions, and cognitive flexibility, but not risk-based decision-making. Psychol. Med. 50, 1-10 (2019).

74. Umbricht, D. et al. Effects of the 5-HT 2A agonist psilocybin on mismatch negativity generation and $A X$-continuous performance task: implications for the neuropharmacology of cognitive deficits in schizophrenia. Neuropsychopharmacology 28, 170-181 (2003).

75. Carter, O. L. et al. Psilocybin links binocular rivalry switch rate to attention and subjective arousal levels in humans. Psychopharmacology 195, 415-424 (2007).

76. Prochazkova, L. et al. Exploring the effect of microdosing psychedelics on creativity in an open-label natural setting. Psychopharmacology $\mathbf{2 3 5}$ 3401-3413 (2018).

77. Griffiths, R. R., Richards, W. A., Johnson, M. W., McCann, U. D. \& Jesse, R. Mystical-type experiences occasioned by psilocybin mediate the attribution of personal meaning and spiritual significance 14 months later. J. Psychopharmacol. 22, 621-632 (2008).

78. Griffiths, R. R., Richards, W. A., McCann, U. \& Jesse, R. Psilocybin can occasion mystical-type experiences having substantial and sustained personal meaning and spiritual significance. Psychopharmacology 187, 268-283 (2006). discussion 284-292.

79. Hartogsohn, I. The meaning-enhancing properties of psychedelics and their mediator role in psychedelic therapy, spirituality, and creativity. Front. Neurosci. 12, 129 (2018).

80. Preller, $\mathrm{K} . \mathrm{H}$. et al. The role of the serotonin $2 \mathrm{~A}$ receptor in the fabric and modulation of personal meaning in lysergic acid diethylamide (LSD)-induced states. Eur. Neuropsychopharmacol. 26, S299-S300 (2016).

81. Kometer, M., Pokorny, T., Seifritz, E. \& Volleinweider, F. X. Psilocybin-induced spiritual experiences and insightfulness are associated with synchronization of neuronal oscillations. Psychopharmacology 232, 3663-3676 (2015).

82. Studerus, E., Kometer, M., Hasler, F. \& Vollenweider, F. X. Acute, subacute and long-term subjective effects of psilocybin in healthy humans: a pooled analysis of experimental studies. J. Psychopharmacol. 25, 1434-1452 (2011).
83. Kraehenmann, R. Dreams and psychedelics: neurophenomenological comparison and therapeutic implications. Curr. Neuropharmacol. 15, 1032-1042 (2017).

84. Lebedev, A. V. et al. Finding the self by losing the self: neural correlates of ego-dissolution under psilocybin. Hum. Brain Mapp. 36, 3137-3153 (2015).

85. Speth, J. et al. Decreased mental time travel to the past correlates with default-mode network disintegration under lysergic acid diethylamide. J. Psychopharmacol. 30, 344-353 (2016).

86. Carhart-Harris, R. L. \& Friston, K. J. The default-mode, ego-functions and freeenergy: a neurobiological account of Freudian ideas. Brain 133, 1265-1283 (2010).

87. Fox, K., Nijeboer, S., Solomonova, E., Domhoff, G. W. \& Christoff, K. Dreaming as mind wandering: evidence from functional neuroimaging and first-person content reports. Front. Hum. Neurosci. 7 (2013).

88. Carhart-Harris, R. \& Nutt, D. Was it a vision or a waking dream? Front. Psychol. 5, 255 (2014).

89. Sanz, C. \& Tagliazucchi, E. The experience elicited by hallucinogens presents the highest similarity to dreaming within a large database of psychoactive substance reports. Front. Neurosci. 12, 7 (2018).

90. Kraehenmann, R. et al. Dreamlike effects of LSD on waking imagery in humans depend on serotonin 2A receptor activation. Psychopharmacology 234, 2031-2046, https://doi.org/10.1007/s00213-017-4610-0 (2017).

91. Vollenweider, F. X. \& Kometer, M. The neurobiology of psychedelic drugs: implications for the treatment of mood disorders. Nat. Rev. Neurosci. 11 642-651 (2010).

92. Ly, C. et al. Psychedelics promote structural and functional neural plasticity. Cell Rep. 23, 3170-3182 (2018).

93. Cole, M. W., Yarkoni, T., Repovš, G., Anticevic, A. \& Braver, T. S. Global connectivity of prefrontal cortex predicts cognitive control and intelligence. J. Neurosci. 32, 8988-8999 (2012).

94. Anticevic, A. et al. The role of default network deactivation in cognition and disease. Trends Cogn. Sci. 16, 584-592 (2012)

95. Douw, L., Wakeman, D. G., Tanaka, N., Liu, H. \& Stufflebeam, S. M. Statedependent variability of dynamic functional connectivity between frontoparietal and default networks relates to cognitive flexibility. Neuroscience $\mathbf{3 3 9}$ 12-21 (2016).

96. Bassett, D. S. et al. Dynamic reconfiguration of human brain networks during learning. Proc. Natl Acad. Sci. USA 108, 7641-7646 (2011).

97. Cole, M. W. et al. Multi-task connectivity reveals flexible hubs for adaptive task control. Nat. Neurosci. 16, 1348-1355 (2013).

98. Monti, R. P. et al. Estimating time-varying brain connectivity networks from functional MRI time series. Neurolmage 103, 427-443 (2014).

99. Hoekzema, E. et al. An independent components and functional connectivity analysis of resting state $\mathrm{fMRI}$ data points to neural network dysregulation in adult ADHD. Hum. Brain Mapp. 35, 1261-1272 (2014).

100. Bressler, S. L. \& Menon, V. Large-scale brain networks in cognition: emerging methods and principles. Trends Cogn. Sci. 14, 277-290 (2010).

101. Berkovich-Ohana, A., Glicksohn, J., Ben-Soussan, T. D. \& Goldstein, A. Creativity is enhanced by long-term mindfulness training and is negatively correlated with trait default-mode-related low-gamma inter-hemispheric connectivity. Mindfulness 8, 717-727 (2017).

102. Jung, R., Mead, B., Carrasco, J. \& Flores, R. The structure of creative cognition in the human brain. Front. Hum. Neurosci. 7 (2013).

103. Rucker, J. J. H., lliff, J. \& Nutt, D. J. Psychiatry \& the psychedelic drugs. Past, present \& future. Neuropharmacology 142, 200-218 (2018).

104. Beck, A. T. Depression: Clinical, Experimental, and Theoretical Aspects (University of Pennsylvania Press, 1967).

105. Nolen-Hoeksema, S., Wisco, B. E. \& Lyubomirsky, S. Rethinking rumination. Perspect. Psychol. Sci. 3, 400-424 (2008).

106. Bouso, J. C., Doblin, R., Farre, M., Alcazar, M. A. \& Gomez-Jarabo, G. MDMAassisted psychotherapy using low doses in a small sample of women with chronic posttraumatic stress disorder. J. Psychoact. Drugs 40, 225-236 (2008).

107. Frecska, E., Bokor, P. \& Winkelman, M. The Therapeutic potentials of ayahuasca: possible effects against various diseases of civilization. Front. Pharmacol. 7, 35 (2016).

108. Allen, M., Poggiali, D., Whitaker, K., Marshall, T. R. \& Kievit, R. A. Raincloud plots: a multi-platform tool for robust data visualization. Wellcome Open Res. 4, 63-63 (2019). 